See discussions, stats, and author profiles for this publication at: https://www.researchgate.net/publication/342708717

\title{
Single Frequency Networks for 5G Broadcast: a Software Defined Radio Experiment
}

Conference Paper · July 2020

CITATIONS

0

6 authors, including:

A. Álvaro Ibáñez Latorre

Universitat Politècnica de València

1 PUBLICATION 0 CITATIONS

SEE PROFILE

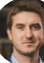

Manuel Fuentes

Fivecomm

30 PUBLICATIONS 218 CITATIONS

SEE PROFILE

Some of the authors of this publication are also working on these related projects:

5G-TOURS View project

Diseño y validación de la segunda generación del estándar de TDT con canal de retorno inalámbrico, para proveer conectividad a Internet en zonas rurales en

Latinoamérica. View project

\section{READS}

300

Borja Inesta

Universitat Politècnica de València

1 PUBLICATION 0 CITATIONS

SEE PROFILE

David Gomez-Barquero

Universitat Politècnica de València

161 PUBLICATIONS 1,891 CITATIONS

SEE PROFILE 


\title{
Single Frequency Networks for 5G Broadcast: a Software Defined Radio Experiment
}

\author{
Alvaro Ibanez*, Borja Inesta*, Manuel Fuentes*, David Gomez-Barquero*, Diarmuid Collins ${ }^{\dagger}$ and Joao F. Santos ${ }^{\dagger}$ \\ *iTEAM Research Institute, Universitat Politecnica de Valencia, Spain \\ ${ }^{\dagger}$ Trinity College Dublin, University of Dublin, Ireland \\ Email:\{alibla, borieher, mafuemue, dagobar\}@iteam.upv.es, \\ $\{$ dcollin5, facocalj\}@tcd.ie
}

\begin{abstract}
Broadcast is a mechanism created for the delivery of the same content to many users simultaneously. This technology has been considered in $3 G P P$ as part of $4 \mathrm{G}$ and recently $5 \mathrm{G}$. One of the key solutions to enhance coverage when using $5 \mathrm{G}$ broadcast is the use of Single Frequency Networks (SFN), where all transmitters deliver the same content at the same frequency in a synchronized manner. This paper is derived from the 5GROSE project, which works towards the transmission of $5 \mathrm{G}$ broadcast services over SFN virtualized networks, using opensource software. The work is divided into three topics. The first part is the development and testing of the first virtualized Multimedia Broadcast Multicast Service over Single Frequency Network (MBSFN) transmission. The second part consists of the introduction of 5G physical layer Release (Rel)-16 specific components. The third part is about the combination of both unicast and broadcast transmissions by means of network slicing. This paper describes in detail the implemented technologies, the testbed facility where these are integrated, as well as the implementation and setup to carry out the experiment.

Index Terms-5G, Single Frequency Network, virtualization, enTV, Software Defined Radio, network slicing
\end{abstract}

\section{INTRODUCTION}

B ROADCAST networks have been traditionally used for the provision of Digital Terrestrial Television (DTT) services. Unlike current mobile cellular services, DTT is delivered by using a High-Power High-Tower (HPHT) topology and Single Frequency Network (SFN) transmitters that are time and frequency synchronized to broadcast the same content simultaneously [1]. Due to the use of Orthogonal FrequencyDivision Multiplexing (OFDM) at the receiver, all transmitted signals and their echoes inside the cyclic prefix $(\mathrm{CP})$ window are coherently combined, enabling large coverage areas with high reliability. Larger areas are translated into less transmitters, which lowers infrastructure and deployment costs.

Another technology providing cost reduction is virtualization [2]. Virtualization is a key element in the definition of the 5G infrastructure [3]. It consists of the creation of software mimicking hardware, enabling network operators to develop and deploy a vast number of different services in an efficient way. Network operators can quickly deliver customized services with virtual machines while reducing the need for expensive hardware. This approach enables the possibility of adding new features or upgrades over time, the scalability in hardware and software, and lowers the management costs by managing all services by software. However, as for parts of the $4 \mathrm{G} / 5 \mathrm{G}$ core virtualization are perfectly adopted, other parts such as the Radio Access Network (RAN) find difficulties to stick to this approach. At the RAN, fast timing requirements limit a full implementation of the functionalities [4].

A good alternative is to combine both unicast and broadcast services by means of network slicing [5]. It is a key concept for future $5 \mathrm{G}$ networks, where the network is divided on slices to offer personalized services for users of different applications and/or verticals, while using the same infrastructure. Even though broadcast services are traditionally offered through HPHT topologies with SFN, they could be combined with unicast also using other configurations, e.g. a Low-Power LowTower (LPLT) topology.

This work describes the setup and implementation of a $5 \mathrm{G}$ broadcast experiment mainly focused on the aforementioned technologies and key enablers. The main objective of the experiment is to create a virtualized Software Defined Radio (SDR)-based SFN infrastructure for 5G broadcast services. The experiment utilizes the IRIS testbed facilities located in Trinity College of Dublin, Ireland [6] and it is based on the srsLTE open-source LTE software implementation [7]. It is divided into three main parts, directly related to the key technologies described. The first part of the experiment consists of the development and testing of a virtualized Multimedia Broadcast Multicast Service over Single Frequency Network (MBSFN) transmission. The second part is based on the 5G LTE-based Terrestrial Broadcast (TB) mode, currently under standardization in Release 16. Some physical layer features that are key in an SFN deployment, such as the use of new numerologies, associated to the Sub-Carrier Spacing (SCS) and $\mathrm{CP}$, are explored. Finally, the third part combines both unicast and broadcast transmissions by means of network slicing. It will be completed as part of the future work.

The document is structured as follows. First, a brief theoretical overview is presented in Section II, including the key aspects for each part of the experiment. The IRIS testbed is presented in Section III, describing the main functionalities and features. The setup and implementation of each of the topics are presented in Section IV. Finally, Section V presents the main conclusions and future work of this experiment. 


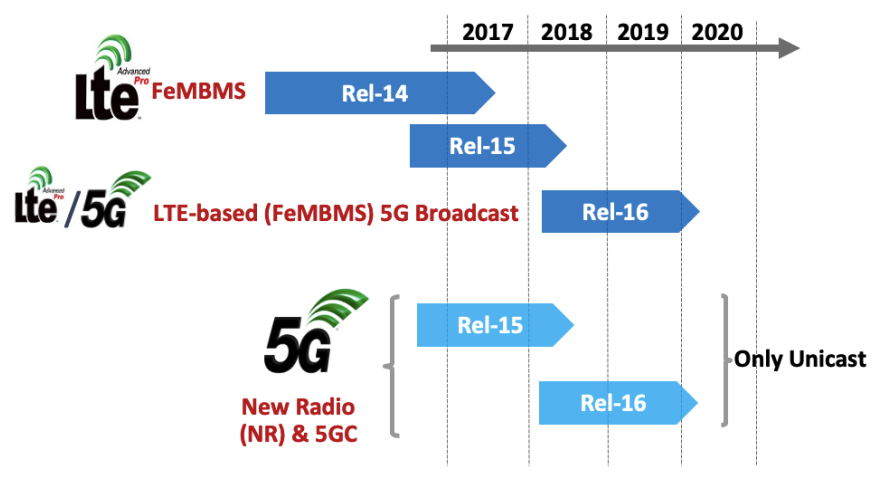

Fig. 1. 5G New Radio (NR) Evolution.

\section{Technology Overview}

This section describes the main innovations and technology components that compose each one of the three parts that form the $5 \mathrm{G}$ broadcast experiment.

\section{A. Single frequency networks}

The deployment of an SFN provides a large number of benefits for $5 \mathrm{G}$ broadcast services. SFN deployments not only increase coverage, but also reduce interference and transmission power [8]. The successful reception within the $\mathrm{CP}$ window also provides higher reliability. Traditionally, DTT networks based on SFN are designed for over-the-top fixed reception and linear systems with Line of Sight (LOS). On the other hand, enhanced TV (enTV) Rel-16 not only aims at keeping this type of transmission with high coverage, but also at reaching a extremely large number of mobile devices in Non-Line of Sight (NLOS) conditions. SFNs will be used in enTV as an alternative topology for content delivery. SFN combined with multicast/broadcast capabilities are a good mechanism when connecting many devices at once, without the need of large infrastructures and taking advantage of the existing infrastructure.

SFNs usually need very stringent synchronization requirements, which involves the use of specific synchronization hardware (e.g. high-precision clocks, Global Positioning System (GPS) reference signals and signal generators) or high computational power in order to implement the synchronization through software, e.g. Precision Time Protocol version 2 (PTPv2) or Synchronous Ethernet (SyncE) [9] [10];

\section{B. 5G Terrestrial Broadcast}

The LTE-based 5G Terrestrial Broadcast (TB) solution, also known as enTV, is currently being standardized by the 3rd Generation Partnership Project (3GPP) within the framework of 5G Rel-16, since currently the 5G Multicast/Broadcast solution has not been standardized in 5G NR - 5G Core (5-GC) [11]. Note that this is an LTE-based technology, and therefore, it is heavily dependent on the Further evolved Multimedia Broadcast Multicast Service (FeMBMS) solution standardized in Rel-14 [12], see Figure 1.
In Rel-14, TB requirements were considered in 3GPP for the first time. The most important features of FeMBMS are the use of a receive-only mode, where there is no need for a SIM card, and hence, allows for an open transmission as used in traditional DTT services; the support of larger Inter-Site Distances (ISD) of up to $60 \mathrm{~km}$, in order to re-use the existing HPHT broadcast infrastructure; and $100 \%$ allocation of radio resources to broadcast services with all subframes within a frame dedicated to the Physical Multicast Channel (PMCH). FeMBMS also includes the cell acquisition subframe (CAS) concept, described in [13].

However, despite the Rel-14 improvements, FeMBMS did not meet all requirements as defined by broadcasters in [14]. Examples are the support for larger ISD, coverage footprints up to $100 \mathrm{~km}$, the support of mobility up to $250 \mathrm{~km} / \mathrm{h}$, and higher data rates. Among the improvements that are currently being included in the on-going Rel-16, new numerologies are included [15]. These numerologies have been designed for HPHT and also Medium-Power Medium-Tower (MPMT) topologies. They are intended for large area roof-top reception and to improve mobility. This includes a CP of $300 \mu s$ with symbol duration of $2.7 \mathrm{~ms}$, and new numerologies with $\mathrm{CP}$ durations of $100 \mu s \mathrm{CP}$ and $400 \mu s$.

\section{Network slicing}

Network slicing is one of the key enabling technologies of 5G. It allows multiple logical networks to be executed as virtually independent commercial operations in a single common physical infrastructure in an efficient and economical way [5]. This is a radical paradigm shift compared to previous generations of wireless networks. The concept was initially proposed for the $5 \mathrm{G}$ core network (5GC) and later evolved to end-to-end (E2E) infrastructures including the RAN. Network slicing allows the configuration of different channels through software within the same physical network. Each of these channels, or slices, can be configured to offer the desired performance in terms of stability, latency, bandwidth or coverage, among other factors.

Nowadays, network slicing is a very interesting option for network operators. In the context of this experiment, a potential alternative is to use two slices, each one for a type of transmission (broadcast and unicast). This option can be very useful for operators, since they can considerably reduce the resources needed to transmit certain content to different users, while also sending specific content to others.

\section{IRIS TESTBED FACILITY}

\section{A. Overview}

The reconfigurable radio IRIS testbed [6] provides a stateof-the-art experimental facility for research and prototyping on future network architectures, radio access technologies and radio design. Figure 2 depicts a high-level overview of the architecture. The facility pairs programmable radio and computational resources with different types of hypervisors to cater to experimenters with multiple types of virtualized network resources and technologies. As a consequence of these 


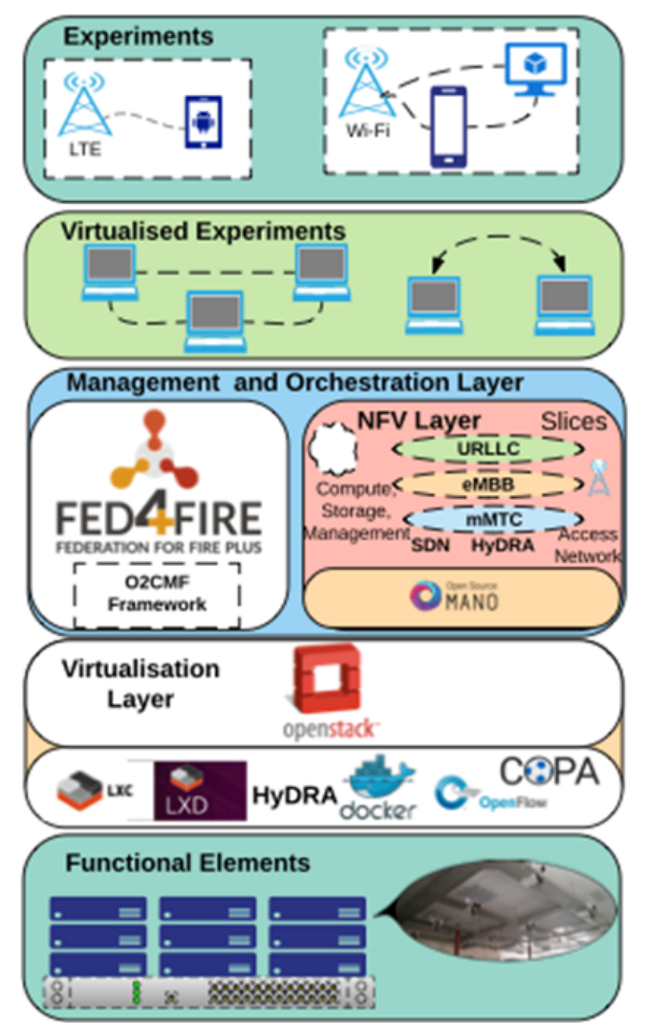

Fig. 2. The IRIS testbed facility and its functional layers.

capabilities, IRIS allows to conduct bleeding-edge experimental research in a myriad of next-generation network topics including those that are part of this work, i.e. network slicing, virtualization and physical layer advancements, as well as investigate their coexistence and integration in real scenarios.

The IRIS testbed consists of five different functional layers, as shown in Figure 2 and described below (from bottom to top).

- Functional Elements Layer: Represents the physical resources including servers, switches and Universal Software Radio Peripherals (USRPs). These elements are sliced by the specific hypervisors on the virtualization layer.

- Virtualization Layer: Exposes slices of physical layer functionalities to applications utilizing a variety of hypervisor tools and technologies, as well as SDR frameworks, including srsLTE [16].

- Management and Orchestration Layer: Supports Open Source Management and Orchestration (OSM) software stack [17], and jFed, which is based on the Slice-based Federation Architecture (SFA) [18].

- Virtualized Experiments Layer: Corresponds to the virtualized testbed resources allocated to the experimenter via OSM or jFed for a predefined period of time.

- Experiments Layer: Refers to the resources directly controlled by the experimenters, sitting on top of the aforementioned layers.

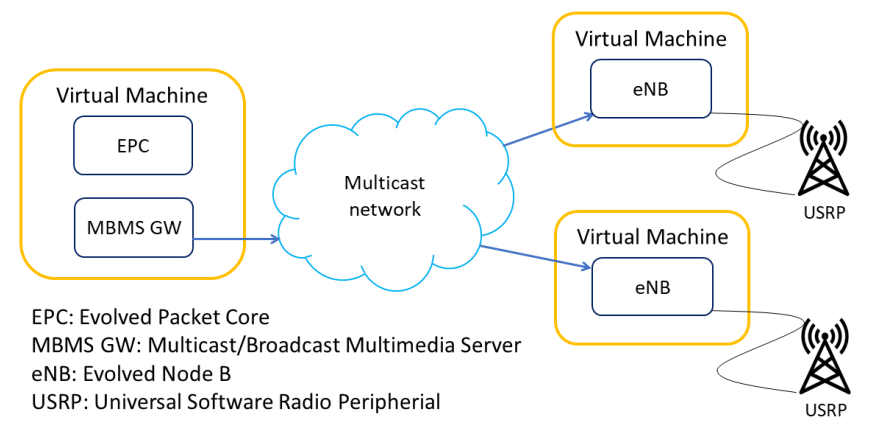

Fig. 3. Virtualized SFN experiment architecture

\section{B. Virtual testbed facility}

The available radio resources at the IRIS facility include over 24 ceiling-mounted Ettus USRP N210s, equipped with SBX daughterboards, supporting frequency ranges between $400 \mathrm{MHz}-4400 \mathrm{MHz}$ with up to $20 \mathrm{MHz}$ of bandwidth. IRIS also offers five Ettus USRP X310s, supporting DC to $6 \mathrm{GHz}$ frequencies, with up to $40 \mathrm{MHz}$ of baseband bandwidth. This equipment is connected to a private computational cloud based on the OpenStack suite of technologies, which can support the deployment of an array of dynamic virtualized computational environments. To expose the functionality of USRPs for applications, IRIS employs a variety of radio hypervisors that freely enable prototyping of wireless systems. These radio hypervisors are instantiated on the OpenStack cloud platform at IRIS. OpenStack at IRIS offers orchestration, fault, service management, and recovery supporting high performance, system availability and redundancy for experimenters.

A fundamental component supporting this malleable environment is a Dell Networking S4048T-ON highperformance SDN/OpenFlow 1.3 enabled switch with $100 \mathrm{M} / 1 \mathrm{G} / 10 \mathrm{G} / 40 \mathrm{GbE}$ interfaces, which is orchestrated by the open-source ONOS network operating system framework [19]. This switch connects USRP radio equipment to single root input/output virtualization (SR-IOV) to dynamically route traffic between USRPs and virtual machine instances. IRIS utilizes ONOS policy-based directives called intents to support actionable operations within the network environment. ONOS intents allow multi-point (physical rack server Ethernet ports) to single point (USRP) flow rules to be installed on the SDN switch.

\section{IMPLEMENTATION AND SETUP}

The following section describes in detail the setup and implementation of the three main components of the $5 \mathrm{G}$ broadcast experiment, i.e. the virtualized SFN, physical layer innovations and network slicing. Note that network slicing is addressed as future work in this paper.

\section{A. Virtualized SFN}

The objective of the first experiment is to develop an SFN inside the srsLTE software by using the IRIS testbed and perform the tests remotely in order to validate it. To 


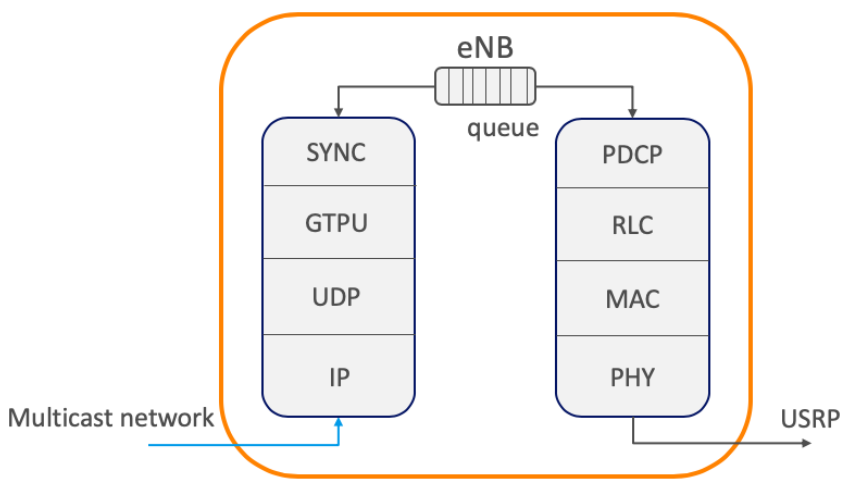

Fig. 4. eNB stack with SYNC protocol.

the best of our knowledge, this experiment is the first one involving virtualized MBSFN transmissions. Figure 3 shows the architecture separating the virtual machines running the srsLTE components and the USRPs used to transmit the frames.

This experiment is divided into three tasks. The first task consists of the selection of synchronisation methods to be used between USRPs and virtual machines. The second task is the implementation of the SYNC protocol, based on [20] and tested in srsLTE. The third and last task is about creating tests to measure the overall SFN performance.

In this work, the radio hardware and the one running the LTE stack are implemented in a separated manner. Therefore, both need to be synchronized. Synchronization is required between USRPs, as well as between virtual machines and containers. To achieve such synchronization, a precision under 1 $\mathrm{ms}$ is needed (which is the subframe duration in both LTE and 5G). An Octoclock-G device from Ettus Research is used to synchronize the USRPs, acting as Remote Radio Head (RRH) at the eNB, in order to select the exact time and frequency to transmit the specific subframes. This device synchronizes time and frequency using GPS and shared Pulse-per-Second (PPS) signals. For the eNBs, two different synchronization approaches were selected. The first approach runs the eNBs using virtual machines with a PTPv2 implementation, i.e. PTPd from sourceforge [21]. The second approach runs the eNBs using containers inside the same virtual machine, sharing the same clock in all eNBs. This approach was proposed to have a reference and compare the results of the synchronisation obtained with PTPv2.

The SYNC protocol implementation was based on srsLTE, in order to develop the SYNC header pack/unpack functions defined in [20]. After developing the packet structure for SYNC PDU type 0, 1 and 3, the next step was to create a multiple consumer-producer thread-safe queue capable of storing the developed SYNC packets. As the technical specification describes, the received multicast data packets need to be stored and sorted by packet number and timestamp when received at the eNB. When the time specified by the

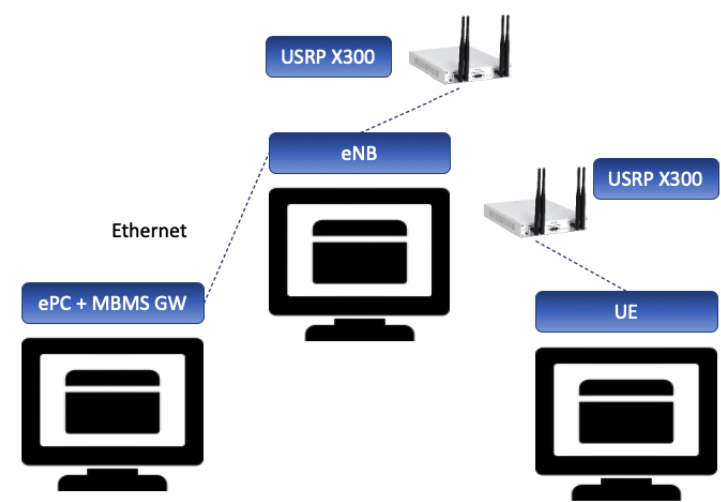

Fig. 5. 5G broadcast experiment setup.

timestamp field in the SYNC packet is reached, the eNB starts transmitting all the SYNC packets with same timestamp. Figure 4 shows the SYNC position in the protocol stack of the eNB and where the developed queue is placed. The last part of this experiment was to configure the experiment in the testbed with the two different architectures in order to see the synchronization obtained using PTPv2 and its effects on MBSFN transmissions.

\section{B. $5 G$ broadcast: new numerologies for larger coverage}

This experiment focuses on the implementation of new OFDM numerologies for 5G broadcast in the srsLTE software. In particular, the two configurations that are included are 7.5 and $1.25 \mathrm{kHz}$. These numerologies were adopted in Rel-12 and Rel-14 respectively with the purpose of establishing a longer $\mathrm{CP}$ and provide larger coverage. Note that the current srsLTE code is Rel-10 compliant. An in-depth study of the code was performed, where all parameters affected by the numerology were considered.

This part of the experiment was split into two implementations. In the first one, the existing srsLTE code was modified accordingly, adding the new numerologies and modifying the functions where these were affecting. Examples are the functions directly related to the PMCH channel. The rest of physical channels were still using a regular $15 \mathrm{kHz}$ SCS. This implementation includes the whole protocol stack (PDCP, MAC, RLC layers). Three virtual machines were used. The setup of the experiment is made up of one VM acting as ePC (MBMS gateway active); one VM acting as eNB, connected to the ePC through an ethernet connection and also connected to a USRP X300; and one VM acting as user, connected to a USRP X300, as Figure 5 shows.

To carry out the tests, the eNB and the UE had to be configured with some physical parameters related to MBMS. Examples are the number of physical resource blocks (PRBs), bandwidth, cell identifiers or number of control symbols. It is also important to add to the routing table the MBMS gateway, in order to start downlink traffic. The network was tested with the iperf software, used to create IP packets and inject traffic through the MBMS gateway. The same software is used at the receiver. 
The second implementation is based on two specific functions for transmission and reception of broadcast content, composed of the PMCH channel and channel filtering. Note that control information is hard-coded. The PMCH information is transmitted using a USRP and received with another. The second USRP receives and decodes the signal, since control information is already known. Note that, despite being an experiment labeled as "5G-Broadcast", just one virtual user has been used. This was considered as a way to carry out the corresponding tests and measurements. In this implementation only two virtual machines are required. Each virtual machine is connected to a USRP X300. In this case, as the control information is already known by both transmitter and receiver, the configuration that can be edited is reduced to a small number of parameters, e.g. are the bandwidth and number of PRBs, or some USRP-related parameters such as transmission and reception gains.

\section{Network slicing}

This section describes the implementation and setup of network slicing, as a future extension of this experiment. It is based on HyDRA, a hypervisor that is key for radio virtualization. It receives raw In-phase and Quadrature (IQ) samples from multiple virtual radio-frequency front-ends and employs software for baseband processing and multiplex into a single transmitted waveform via USRP. Despite its advantages, such as agnostic multiplexing to support different technologies or the little overhead it adds, HyDRA also has a series of drawbacks that affect the design and implementation of this experiment. The most important disadvantage is that HyDRA is not able to cope with the strict timing requirements of upper layers, such as MAC or PDCP layers. Therefore, HyDRA does not allow a full stack implementation as described in LTE, just allowing the use of a specific physical channel. For this reason, specific files for the transmitted channels are needed. Transmissions of the physical data channel, Physical Downlink Share Channel (PDSCH) will be established in parallel with transmissions of the $\mathrm{PMCH}$.

\section{CONCLUSiOnS AND Future Work}

This work is derived from the 5G-ROSE project, and consists of the implementation of a virtualized SFN for the transmission of $5 \mathrm{G}$ broadcast services in a SDR laboratory environment. The implementation uses the IRIS testbed facility in Trinity College of Dublin (Ireland) and it is based on the srsLTE open-source software. The work is divided into three parts, i.e. the development and testing of the first virtualized MBSFN transmission; the introduction of physical layer Rel-16 components; and as part of our future work, the implementation of network slicing for the simultaneous transmission of both unicast and multicast content. The paper described in detail the implemented technologies as well as the setup to carry out the experiment. It may become an important milestone for the SDR implementation of SFN and $5 \mathrm{G}$ broadcast technologies.

\section{ACKNOWLEDGEMENTS}

This work has received funding from the European Horizon 2020 Program project Orchestration and Reconfiguration Control Architecture (ORCA), in the third Open Call for experiments, under Grant Agreement no. 732174. This work was partially supported by the H2020 5G-TOURS European project (Grant Agreement No. 856950).

\section{REFERENCES}

[1] M. Liang, W. Chen and B. Long, "OFDM Timing Synchronization Schemes in SFN Channels," 2007 2nd IEEE Conference on Industrial Electronics and Applications, Harbin, 2007, pp. 1535-1538, doi: 10.1109/ICIEA.2007.4318664.

[2] J. F. Santos, M. Kist, J. van de Belt, J. Rochol and L. A. DaSilva, "Towards Enabling RAN as a Service - The Extensible Virtualisation Layer," ICC 2019 - 2019 IEEE International Conference on Communications (ICC), Shanghai, China, 2019, pp. 1-6, doi: 10.1109/ICC.2019.8761679.

[3] M. Kist, J. Rochol, L. A. DaSilva and C. B. Both, "SDR Virtualization in Future Mobile Networks: Enabling Multi-Programmable Air-Interfaces," 2018 IEEE International Conference on Communications (ICC), Kansas City, MO, 2018, pp. 1-6, doi: 10.1109/ICC.2018.8422643.

[4] CISCO Public, "Reimagining the End-to-End Mobile Network in the 5G Era", 2019.

[5] I. d. Silva, "Impact of Network Slicing on 5G Radio Access Networks" EUCNC, Athens, June 2016.

[6] Trinity College Dublin, "IRIS testbed Home Page". Available: http://iristestbed.eu/.

[7] Software Radio Systems, "SRS Home Page" . Available: https://www.softwareradiosystems.com/.

[8] D. Plets, W. Joseph, L. Verloock, L. Martens, P. Angueira and J. A. Arenas, "SFN gain in broadcast networks," 2011 IEEE International Symposium on Broadband Multimedia Systems and Broadcasting (BMSB), Nuremberg, 2011, pp. 1-6.

[9] M. Saily, C. Barjau, D. Navratil, A. Prasad, D. Gomez-Barquero, F. Tesema. "5G radio access networks: Enabling efficient point-tomultipoint transmissions," IEEE Vehicular Technology Magazine 14 (4), 29-37. April, 2020.

[10] ; M. Säily, C.B. Estevan, J.J. Gimenez, F. Tesema, W. Guo, D. GomezBarquero. " $5 \mathrm{G}$ radio access network architecture for terrestrial broadcast services", IEEE Transactions on broadcasting. September, 2019.

[11] D. Gomez-Barquero, D. Navratil, S. Appleby, M. Stagg: "Point-toMultipoint Communication Enablers for the Fifth Generation of Wireless Systems," IEEE Communications Standards Magazine 2(1): 53-59 (2018).

[12] J. J. Gimenez, J. L. Carcel, M. Fuentes, E. Garro, S. Elliott, D. Vargas, C. Menzel, D. Gomez-Barquero: "5G New Radio for Terrestrial Broadcast: A Forward-Looking Approach for NR-MBMS," TBC 65(2): 356-368 (2019).

[13] D. Gomez-Barquero, J.J Gimenez, and R. Beutler "3GPP Enhancements for Television Services: LTE-based 5G Terrestrial Broadcast," Wiley Encyclopedia of Electrical and Electronics Engineering, May, 2020.

[14] 3GPP TR 38.913, v15.0.0, "Study on scenarios and requirements for next generation access technologies", July, 2018.

[15] 3GPP TR 36.976, v16.0.0, "Overall description of LTE-based 5G broadcast (Release 16)", April, 2020.

[16] I. Gomez-Miguelez, et al. "srsLTE: an open-source platform for LTE evolution and experimentation." Proceedings of the Tenth ACM International Workshop on Wireless Network testbeds, Experimental Evaluation, and Characterization. 2016.

[17] OSM, Available: https://osm.etsi.org/

[18] J. Auge, and T. Friedman. "The open slice-based facility architecture (open sfa).", 2012.

[19] Open Network Operating System (ONOS), Available: https://www.opennetworking.org/onos/

[20] 3GPP TS 25.446, v14.0.0, "MBMS synchronisation protocol (SYNC)", March, 2017.

[21] PTPd, Available on sourceforge: https://sourceforge.net/projects/ptpd/ 